\title{
Fisiognomica a Qumran: a proposito di 4Q186
}

\author{
Alessandro Catastini* \\ Università di Roma «La Sapienza»
}

Physiognomics IN Qumram: Concerning 4Q186.- In a previous study, the Author singled out physiognomonic practice by Essenes according to Flavius Josephus' source in Ant II, 119-161. In this article, the research is extended to the Qumranic manuscript 4Q186, a parchment carrying a text whose content is manifestly physiognomonic. In consequence of a comparison between this text and the so-called Two Spirits Treatise of 1QS, the Author argues that the expression רוח לו, utilised in 4Q186, points out the "typology" of the spirit of the individuals that are considered in this text.

KeYwords: Qumran; Physiognomy; 4Q186; Two Spirit Treatise; Cryptic Manuscript.

FisIogNómica EN QuMRÁn: A PROPÓSITO DE 4Q186.- En un estudio previo, el autor había estudiado la práctica fisiognómica de los esenios a partir de la evidencia recogida por Flavio Josefo (Ant II, 119-161). En esta ocasión, la investigación se extiende al manuscrito 4Q186, cuyo texto tiene un contenido claramente fisiognómico. La comparación entre este texto y el denominado Tratado de los Dos Espíritus (1QS), lleva al autor a señalar que la expresión רוח לו utilizada en 4Q186 muestra la «tipología» del espíritu de los individuos de los que trata este texto.

Palabras Clave: Qumrán; fisiognomía; 4Q186; Tratado de los Dos Espíritus; manuscrito críptico.

In uno studio precedente ${ }^{1}$ ho preso in esame le affinità tra l'essenismo e un'ulteriore esperienza di tipo comunitario, quella pitagorica. L'accostamento tra i due fenomeni, come è noto, risale già allo stesso Flavio Giuseppe sia nella

*alessandro.catastini@uniroma1.it

${ }^{1}$ «Flavio Giuseppe e la filosofia degli Esseni», in A. Hilhorst, É. Puech, E. TigchelaAR (eds.), Flores Florentino. Dead Sea Scrolls and Other Early Jewish Studies in Honour of Florentino García Martínez (Leiden - Boston 2007), pp. 53-62. Di recente, cfr. anche J. TAYLOR, Pythagorean and Essenes: Structural Parallels (Louvain 2004), il quale ritiene che i paralleli strutturali tra i modi di vita di Pitagorici ed Esseni siano da attribuire a contatti reciproci, in particolare per l'influenza dei primi sui secondi: Alessandria sarebbe stato il canale di comunicazione in tal senso (p. 106). Altri lavori da ricordare sul tema: E. J. C. TigchelaAR, «The White Dress of the Essenes and the Pythagoreans», in F. García Martínez, G. P. Luttikhuizen (eds.), Jerusalem, Alexandria, Rome. Studies in Ancient Cultural Interaction in 
sua formulazione esplicita (Ant XV, 371), sia nei dati che lo storico espone nella sua lunga descrizione degli Esseni (Bell II, $\S \S 119-161$ ). Nel mio studio ho in particolare voluto sottolineare le affinità concernenti i criteri di ammissione alle comunità pitagorica da un lato e a quella essena, secondo la descrizione di Giuseppe, dall'altro.

In merito all'esperienza pitagorica mi è parso singolarmente utile il passo di Giamblico in De vita Pythagorica I, XVII, 71-74. Qui Giamblico trasmette una fonte risalente a Timeo di Tauromenio (IV-III sec. a.C.) ${ }^{2}$ che descrive una sintesi fra i «tratti (

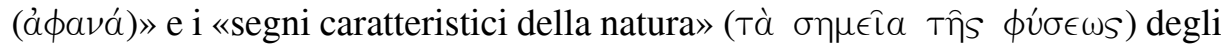
stessi, i quali sono invece visibili ( $\phi \propto \nu \in \rho a ́)$. Il passo che illustra la relazione tra i due concetti è di per sé eloquente ed esplicativa:

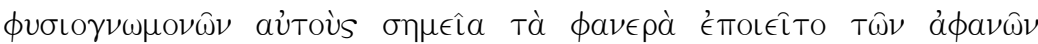

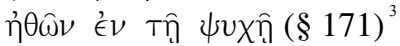

È di notevole rilevanza che il termine $\hat{\jmath} \theta$ os, qui utilizzato nel significato di «caratteristiche esteriori», compaia nel trattato pseudoaristotelico $\Phi v \sigma \iota \gamma \nu \omega \mu \omega \nu \iota \kappa a ́$ proprio con questo senso; ${ }^{4}$ anche Flavio Giuseppe usa il termine con questa accezione in Bell II, 138:

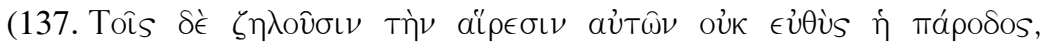

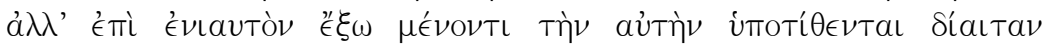

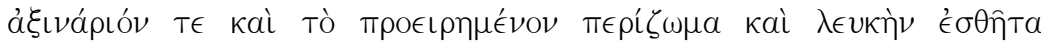

Honour of A. Hilhorst (Leiden - Boston 2003), pp. 301-321; A. BerLeJung, «Physiognomik im Alten Testament, den Apokryphen und Qumran», Leqach 6 (2005), pp. 7-25. Sull'argomento fisiognomico in ambito siriaco: P. G. Borbone, «Un capitolo siriaco di fisiognomica tra gli Aneddoti divertenti di Bar Hebraeus», EVO 25 (2002), pp. 189-202.

${ }^{2}$ Cfr. Giamblico, La vita pitagorica, introduzione, traduzione e note di Maurizio Giangiulio (Milano 1991), p. 203; Jamblique, Vie de Pythagore. Introduction, traduction et notes par L. Brisson et A. Ph. Seggonds (Paris 1996), p. 170; G. StaAb, Pythagoras in der Spätantike. Studien $z u$ De Vita Pythagorica des Iamblichos von Chalkis (München - Leipzig 2002), pp. 306-307.

${ }^{3}$ «Analizzandone [scil. dell'iniziando] la fisiognomica, per mezzo dei tratti fisici [Pitagora]

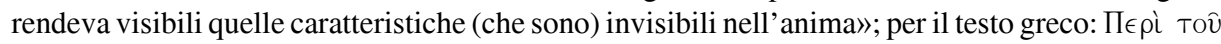

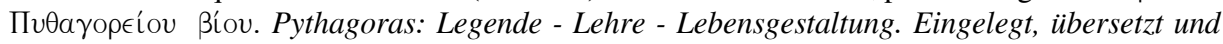
mit interpretierenden Essays versehen von Michael von Albrecht, John Dillon, Martin George, Michael Lurje, David S. du Toit (Stuttgart 2002).

${ }^{4}$ Cfr. Pseudo Aristotele, Fisiognomica - Anonimo Latino, Il trattato di fisiognomica

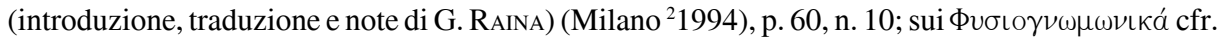
di recente, L. ARATA, «I due trattati di physiognomica attribuiti ad Aristotele», RivCultClassMediev 51 (2009), pp. 11-38. 


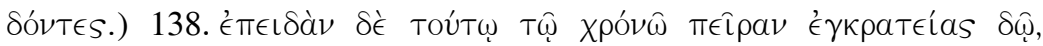

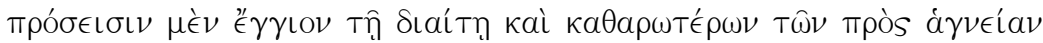

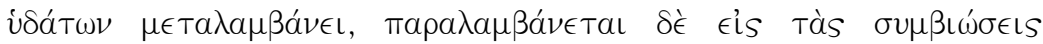

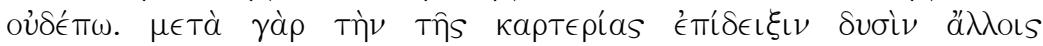

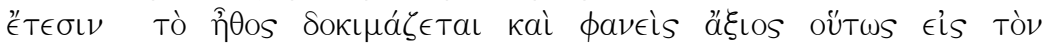

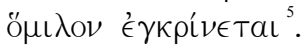

In questo passo riscontriamo un parallelismo con i «segni caratteristici della natura» (il termine è $\sigma \hat{\mu} \mu \in \mathrm{L} \nu$ ) della fonte riportata da Giamblico. Giuseppe afferma che per l'ammissione alla comunità degli Esseni l'esame

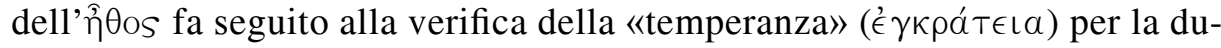

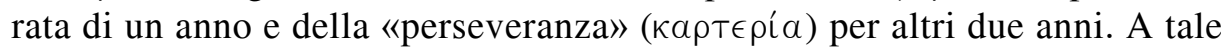
proposito rilevo che la mancanza di una precisazione temporale per l'esame dell' $\jmath$ os «ne denuncia la diversità dalle altre due in merito alla modalità poiché l'esame dell' dato. Piuttosto, è da supporre l'intervento di uno specifico esaminatore -il cui compito è da vedere descritto nell'uso del verbo che si può per l'appunto

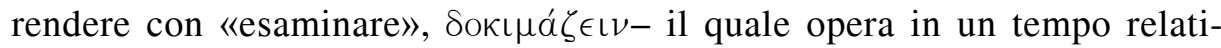
vamente breve per interpretare quei tratti fisiognomici che anche qui sono

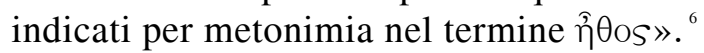

Il dettaglio fisiognomico, in sé, non costituisce ovviamente un dato che permetta di identificare gli Esseni con i Pitagorici: semmai testimonia il fenomeno di un contatto dei primi con la filosofia dei secondi. In un contesto di analisi più generale -come osservò a suo tempo M. H. Goshen-Gottstein riguardo all'identificazione essena dei testi di Qumran ${ }^{7}$ - potrebbe addirittura esser considerato

${ }^{5} \ll(137$. A chi desidera far parte della loro setta non viene concesso di entrare immediatamente, ma lasciandolo fuori per un anno gli fanno seguire la stessa norma di vita, dandogli una piccola scure e la predetta fascia per i fianchi e una veste bianca.) 138. Dopo che in questo periodo di

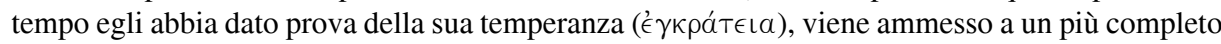
esercizio della regola e ottiene acque più pure per la purificazione, ma non ancora è introdotto

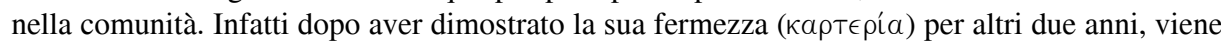

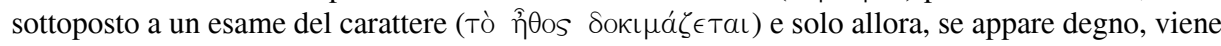
ascritto alla comunità».

${ }^{6}$ CAtastini, «Flavio Giuseppe e la filosofia degli Esseni», p. 60. Alla bibliografia generale sulla fisiognomica che ho citato nel mio contributo è adesso da aggiungere: M. C. PARsons, Body and Character in Luke and Acts: the Subversion of Physiognomy in Early Christianity (Grand Rapids, MI 2006); S. Swain (ed.), Seeing the Face, Seeing the Soul. Polemon's Physiognomy from Classical Antiquity to Medieval Islam. With chapters by George Boys-Stones, Jas Elsner, Antonella Ghersetti, Robert Hoyland, Ian Repath, and Simon Swain (Oxford 2007).

${ }^{7}$ M. H. Goshen-Gottstein, «Anti-Essene Traits in the Dead Sea Scrolls», VT 4 (1954), pp. 141-147. 
un tratto anch'esso autonomamente comune di più organizzazioni comunitarie. Tuttavia, in questo caso ritengo possibile procedere ulteriormente, ampliando la ricerca all'interno dei testi del Mar Morto. La legittimità d'una tale comparazione -ovvero tra i Pitagorici e la comunità di cui si parla nella Regola- è giustificata soprattutto da due motivi: la presenza di testi che si possono facilmente ricondurre alla pratica dell'esame fisiognomico e le testimonianze di procedure di ammissione a una comunità.

I testi che manifestano un marcato carattere «fisiognomico» sono 4Q186, 4Q534 e 4Q561 ${ }^{8}$ e di particolare interesse si rivela il primo di essi, il quale presenta numerosi aspetti che hanno attratto l'attenzione degli studiosi. Anzitutto, esso riproduce un testo composto parzialmente in maniera cosiddetta «criptica»: infatti, assieme alla scrittura ebraica, in caratteri sia quadrati sia arcaici, convive il greco e talora l'andamento della scrittura prosegue in maniera che è inversa per l'ebraico, da sinistra a destra. Il contenuto riguarda la forma di parti del corpo di alcuni soggetti presi in esame: poiché il testo è frammentario, non si può dire con certezza quale fosse il numero di costoro. La lacunosità del manufatto concorre purtroppo a mantenere a prima vista oscuri alcuni altri particolari: un riferimento all'impurità (טמא: fr. 1, col. II, r. 1), a una misteriosa «pietra di granito» (אבן צונם: fr. 1, col. II, r. 2), a non meglio identificati «segreti» (נצרנו[ת: fr. 1, col. II, r. 4), a qualcosa di «spaventoso» (מיראות, fr. 1, col. III, r. 3). Altri particolari hanno fatto pensare alla presenza di una componente astrologica: il termine «colonna» (עמוד: fr. 1, col. II, r. 7), da taluni interpretato nel senso di «costellazione», ${ }^{9}$ la menzione, per due

${ }^{8}$ Su questi manoscritti è stata prodotta un'ampia bibliografia; per le singole editiones principes, si veda F. García Martínez (a cura di), Testi di Qumran, edizione italiana a cura di C. Martone (Brescia 1996), pp. 742-743 (4Q186), 766 (4Q534), 768 (4Q561). Per la bibliografia, si veda quella raccolta in M. Popović, Reading the Human Body. Physiognomics and Astrology in the Dead Sea Scrolls and Hellenistic-Early Roman Period Judaism (Leiden - Boston 2007). In particolare, su 4Q186 quale testo con cui si esamina un iniziando: Ph. S. AlEXANDER, «Physiognomy, Initiation, and Rank in the Qumran Community», in H. CANCIK, H. LichtenBerger, P. SCHÄFER (eds.), Geschichte-TraditionReflection. Festschrift für Martin Hengel zum 70. Geburtstag. Band 1: Judentum (Tübingen 1996), pp. 385-394: 391; A. Lange, «The Essene Position on Magic and Divination», in M. Bernstein, F. García Martínez, J. Kampen (eds.), Legal Texts and Legal Issues. Proceedings of the Second Meeting of the International Organization for Qumran Studies, Cambridge 1995. Published in Honour of Joseph M. Baumgarten (STDJ 23) (Leiden 1997), pp. 387-390: 389-390; Ph. S. AleXANDER, «6. I rotoli del Mar Morto. a) 4 QCriptyc (4 Q186)», in E. SchüRer, Storia del popolo giudaico al tempo di Gesù Cristo (175 a.C - 135 d.C.), III.1 (Brescia 1997), pp. 474-479: 475; J. R. DAVILA, «4QMess Ar (4Q534)», DSD 5 (1998), pp. 369-370.

${ }^{9} \mathrm{Cfr}$. M. Delcor, «Recherches sur un horoscope en langue hébraïque provenant de Qumran», $R Q 20$ (1966), pp. 521-542: 529. 
volte, del «toro» (שור: in entrambi i casi, al fr. 1, col. II, r. 9), nell' un caso con l'espressione «nel piede del toro» (ברגל השור: fr. 1, col. II, r. 9) in riferimento al momento di una «nascita» (מולד: fr. 1, col. II, r. 8; fr. 2, col. I, rr. 4, 8), nell'altro come «animale» (בהמה: fr. 1, col. II, r. 9; in fr. 2, col. I, r. 9 ricorre di nuovo il termine, ma le lacune non permettono di sapere quale fosse l'animale) da connettere al soggetto preso in esame. È peraltro sintomatico come la combinazione dei vari elementi ricordati conducano alla formulazione di una sorta di destino dell'esaminato: «sarà povero», viene infatti sentenziato (עיה עני: fr. 1, col. II, r. 9).

Infine, è da ricordare quella che costituisce probabilmente l'informazione più importante rimastaci del documento: il richiamo a uno «spirito» (רוח) dell'individuo preso in esame. In ciò che rimane del testo, vi viene fatto riferimento tre volte (fr. 1, col. II, rr. 7-8; fr. 1, col. III, rr. 5-6; fr. 2, col. I, rr. 6-8) ed è descritto per le parti di luce o di tenebra che esso può avere. Il computo finale di queste parti corrisponde al numero nove e in base alle loro proporzioni viene definita, su base maggioritaria, l'appartenenza dell'esaminato e quindi la sua idoneità a una eventuale ammissione.

Le varie interpretazioni di questo testo sono riconducibili ad alcuni puntichiave: il legame tra astrologia e fisiognomica da un lato, l'accostamento alla cosiddetta «Dottrina dei Due Spiriti» dall'altro. ${ }^{10}$

In 4Q186 viene esposto un metodo di esame nel quale le componenti astrologica e fisiognomica sono tra loro in stretta relazione. Sin dai primi studi dedicati al manoscritto non si è mancato di sottolineare questo aspetto, collegandolo alla questione del predeterminismo e del dualismo di Qumran. ${ }^{11}$ In

${ }^{10}$ In particolare, si sono dichiarati favorevoli all'accostamento: H. STEGEMANN, «Zu Textbestand und Grundgedanken von 1QS III, 13-IV, 26», RQ 13/49-52 (1988), pp. 95-131: 126; Alexander, «6. I rotoli del Mar Morto»; Alexander, «Physiognomy, Initiation, and Rank»; G. J. BrooKe, «Body Parts in Barkhi Nafshi and the Qualifications for Membership of the Worshipping Community», in D. K. Falk, F. García Martínez, E. M. Schuller (eds.), Sapiential, Liturgical and Poetical texts from Qumran: Proceedings of the Third Meeting of the International Organization for Qumran Studies, Oslo 1998, Published in Memory of Maurice Baillet (Leiden 2000), pp. 79-94: degli ultimi tre studi si tratterà ampiamente più oltre.

${ }^{11}$ Cfr. Delcor, «Recherches», p. 533 ; Alexander, «6. I rotoli del Mar Morto», p. 474; Alexander, «Physiognomy, Initiation, and Rank», p. 389; F. García Martínez, «Magic in the Dead Sea Scrolls», in J. R. Bremmer and J. R. Veenstra (eds.), The Metamorphosis of Magic from Late Antiquity to the Early Modern Period (Leuven 2002), pp. 14-33 = ID., Qumranica Minora II: Thematic Studies in the Dead Sea Scrolls (Leiden 2007), pp. 109130: $128-129$. 
particolare F. Schmidt ${ }^{12}$ e M. Albani ${ }^{13}$ hanno formulato in proposito delle teorie piuttosto articolate. Il primo isola in cinque componenti la struttura del metodo d'esame applicato in 4Q186: 1 . descrizione fisica del soggetto; 2 . indicazione della «colonna» (עמוד) cui questi appartiene; 3. descrizione spirituale sulla base delle parti di luce e di tenebra; 4. segno zodiacale; 5 . predizione sulla condizione sociale. ${ }^{14}$ Secondo Schmidt l'oroscopo veniva determinato sulla base del concepimento: l'opinione è conseguente all'interpretazione del termine מולד quale forma hofal (《generato») e al collegamento con un metodo oroscopico «caldaico» riportato da Censorino (III sec. d.C.). ${ }^{15}$ Il riferimento al «piede del toro» riguarderebbe pertanto il momento in cui l'individuo fu concepito e non quando nacque. A partire da questo dato, Schmidt passa poi a definire le parti di luce e di tenebra sulla base dei sistemi zodiacali della tradizione alessandrina. ${ }^{16}$ In tal senso, vien fatto riferimento alla rappresentazione di un cerchio zodiacale attestato nei papiri oroscopici greci con direzione oraria, mentre lo è in direzione antioraria in alcuni mosaici delle sinagoghe di Galilea; il cerchio è diviso in quattro quadranti, corrispondenti alle quattro stagioni, ove il primo quadrante corrisponde al solstizio d'inverno, che ha inizio con il Capricorno. I segni zodiacali sono ripartiti in due gruppi, o «parti»: i segni che seguono il sole, o «segni diurni», e quelli che seguono la Luna, o «segni notturni»; il sole e i segni diurni sono predominanti in primavera ed estate, la luna e i segni notturni lo sono in autunno e inverno. Ogni segno zodiacale viene diviso in tre decadi (quindi, approssimativamente un mese): ne consegue che il sole, nel suo percorso annuale, attraversa 36 decadi corrispondenti ai dodici segni. Queste decadi si suddividono a loro volta in 18 decadi diurne e 18 notturne; l'oroscopo sarà determinato dal decano in cui sia da situarsi il

${ }^{12}$ F. Schmidt, «Astrologie juive ancienne: Essai d'interprétation de 4QCryptique (4Q186)», RQ 18/69 (1997), pp. 125-141; ID., «אסטרולוגיה וגירה קדומה בקומראן", Qadm 30 (1997), pp. 115118; ID., «Ancient Jewish Astrology. An Attempt to Interpret 4QCryptic (4Q186)», in M. E. SToNE, E. G. Chazon (eds.), Biblical Perspectives: Early Use and Interpretation of the Bible in the Light of the Dead Sea Scrolls. Proceedings of the First International Symposium of the Orion Center for the Study of the Dead Sea Scrolls and Associated Literature, 12-14 May, 1996 (Leiden 1998), pp. 189-205.

${ }^{13}$ M. Albani, «Horoscopes in the Qumran Scrolls», in P. W. Flint and J. C. VanderKam (eds.), The Dead Sea Scrolls After Fifty Years. A Comprehensive Assessment, II (Leiden - Boston Köln 1999), pp. 279-330; M. Albani, «Horoscopes», in L. H. Schiffman, J. C. VanderKam (eds.) Encyclopedia of the Dead Sea Scrolls (Oxford 2000), pp. 370-373.

${ }^{14}$ SCHMIDT, «Astrologie juive ancienne», p. 126.

${ }^{15}$ Schmidt, «Astrologie juive ancienne», pp. 130-132, con rimando ad A. Bouché-LeCLerCQ, L'Astrologie grecque (Paris 1899), p. 377.

${ }^{16}$ Bouché-LeClercQ, L'Astrologie grecque, pp. 155-157. 
momento del concepimento; da questo fino al momento della nascita verranno contate 27 decadi, corrispondenti ai mesi della gestazione $(3 \times 9)$. In questo modo si spiegherebbero le parti di luce e di tenebra che vengono attribuite all'individuo e perché queste parti siano in totale di nove."

A seguito della sua ricostruzione, Schmidt osserva che 4Q186 parrebbe riflettere una sorta di «dualismo relativo», poiché basato sul rapporto della distribuzione di luce e di tenebra all'interno di un insieme di nove parti; poiché ciò non collimerebbe con il dualismo assoluto degli Esseni di Qumran, Schmidt conclude che i testi oroscopici di Qumran non sarebbero da riferire alla comunità. ${ }^{18}$

Albani, dal canto suo, vede nel «piede del toro» l'ascendente del soggetto preso in esame ed è partendo dalla posizione dell'ascendente al momento della nascita che si spiegherebbero le parti di luce e di oscurità: nella fattispecie, sei di esse si troverebbero al di sopra dell'orizzonte mentre tre sarebbero ancora al di sotto. ${ }^{19}$ Come già Schmidt, anche Albani ritiene che i testi oroscopici non riflettano una pratica in uso presso la comunità di Qumran, ma che la loro presenza nel sito fosse dovuta alla necessità di disporre di un documento di una dottrina -l'astrologia- cui gli Esseni si sarebbero opposti. ${ }^{20}$

La teoria di Albani ha avuto una elaborazione ulteriore a opera di M. Popović: rigettando la teoria di Schmidt ${ }^{21}$ e accogliendo nella sostanza quella di Albani, ${ }^{22}$ questi ritiene che 4Q186 testimoni l'uso divinatorio della fisiognomica per determinare l'oroscopo e la natura dello «spirito zodiacale» di una persona, affermando che solo come probabilità il riferimento alle parti di luce e di tenebra poterono essere interpretate a Qumran secondo le concezioni dualistiche dei testi settari, ma «it is not necessary, nor desirable, to explain this terminology only from that perspective». ${ }^{23} 4 \mathrm{Q} 186$ non sarebbe pertanto un testo settario, ma farebbe parte di un «catalogo» fisiognomico vòlto a stabilire l'ascendente del segno zodiacale e la relativa suddivisione in parti di luce e di tenebra sulla

\footnotetext{
${ }^{17}$ SCHмIDT, «Astrologie juive ancienne», pp. 132-133.

${ }^{18}$ SснмidT, «Astrologie juive ancienne», p. 139. Di contro, sulla presenza di un dualismo «relativo» si sono convincentemente espressi: P. WERnBERG MøLler, The Manual of Discipline (Leiden 1957), p. 67, n. 43; H. Lichtenberger, Studien zum Menschenbild in Texten aus der Qumrangemeinde (Göttingen 1980), p. 148; STEGEMAnN, «Zu Textbestand», p. 97.

${ }^{19}$ Albani, «Horoscopes in the Qumran Scrolls», pp. 305-309.

${ }^{20}$ Albani, «Horoscopes in the Qumran Scrolls», p. 313.

${ }^{21}$ Popović, Reading the Human Body, pp. 152-155.

${ }^{22}$ Popović, Reading the Human Body, pp. 155-163.

${ }^{23}$ Popović, Reading the Human Body, p. 131.
} 
base dell'esame fisiognomico. ${ }^{24}$ La negazione del dualismo operante nello spirito dell'uomo permette altresì allo studioso di introdurre un nuovo concetto per il termine רוח, derivante da una riflessione sull'effettivo significato dell'espressione רוח לו, ricorrente in fr. 1, col. II, r. 7; col. III, r. 4; fr. 2, col. I, r. 6 (ove la ו di bè in lacuna): «there is a spirit for him» è l'interpretazione, ${ }^{25}$ desumendone che vi si faccia riferimento a uno spirito che non è quello interiore dell'individuo esaminato, bensì uno spirito esterno. Lo «spirito» di 4Q186 sarebbe in relazione col segno zodiacale e quindi la costruzione רוח לו non sarebbe da riferire allo spirito umano, bensì a quello zodiacale; ${ }^{26}$ uno spirito che, peraltro, influenzerebbe la morfologia del corpo.

Non s'intende qui entrare nel merito della questione astrologica, ossia quale sia stato il metodo zodiacale utilizzato dall'autore del testo di 4Q186; occorre nondimeno osservare che, nei suoi punti fondamentali, l'ipotesi di Popović è רוח לו facilmente contestabile. La particolare interpretazione dell'espressione poggia sull'aprioristico rifiuto di un qualsivoglia collegamento con il dualismo espresso in 1QS. Tuttavia, è proprio grazie a un'attenta lettura di quest'ultimo testo che tale opinione deve essere rigettata. Popović ritiene che con רוח לו si intenda richiamare uno spirito esterno all'esaminando; qualora ci si fosse voluti riferire al suo spirito interiore, argomenta Popović, sarebbe stata impiegata la forma רוחו.

D'altro lato, riescono a questo punto particolarmente istruttivi i precedenti contributi di Ph. S. Alexander ${ }^{27}$ e G. J. Brooke. ${ }^{28}$

Il primo ha pertinentemente definito 4Q186 un «testo di fisiognomica astrologica» sottolineando la stretta relazione di complementarietà fra le due componenti, ${ }^{29}$ avanzando l'ipotesi che servisse per l'ammissione a una setta; ${ }^{30}$ inoltre, proprio a tale scopo il testo sarebbe volutamente criptico: poiché rivolto agli esperti e precluso agli altri.

\footnotetext{
${ }^{24}$ Popović, Reading the Human Body, pp. 170-171.

${ }^{25}$ Popović, Reading the Human Body, p. 193.

${ }^{26}$ Popovič, Reading the Human Body, pp. 202-204.

${ }^{27}$ AleXAnder, «6. I rotoli del Mar Morto»; AleXAnder, «Physiognomy, Initiation, and Rank».

${ }^{28}$ BRooke, «Body Parts».

${ }^{29}$ AleXANDER, «6. I rotoli del Mar Morto», p. 474; AleXAndER, «Physiognomy, Initiation, and Rank», pp. 385-386.

${ }^{30}$ AleXander, «6. I rotoli del Mar Morto», p. 475; AleXander, «Physiognomy, Initiation, and Rank», p. 391.
} 
Brooke ha mostrato come i «segni» (אותות) di cui si parla in 1QS III 15 siano da riferire alle caratteristiche fisiche degli uomini ${ }^{31}$, mettendo in rilievo l'importanza che queste hanno all'interno degli inni 4Q434 e 4Q436, appartenenti al genere $B \bar{a} r^{e} k \hat{\imath} N a f \hat{s} \hat{\imath}$, specialmente se messi in relazione (85-86) con le norme di integrità fisica richiesta ai componenti della comunità: norme che sono esplicitamente esposte nella Regola della Congregazione (1QSa II, 3-9), nella Regola della Guerra (1Q11 VII, 4-5) e in una delle copie qumraniche del Documento di Damasco (CD XV, 15-17).

Le posizioni di Alexander e Brooke procedevano in direzione opposta a quelle che Popović avrebbe elaborato e questi, in particolare, ha controbattuto principalmente il primo dei due, contestandogli la legittimità dell'accostamento di 4Q186 alla «Dottrina dei Due Spiriti»:

Without the context of the Two Spirits Treatise there seems to be no reason to interpret the light and darkness terminology of 4QZodPhys [scil. 4Q186] as dualistic. There are no clues in the extant text that the «house of light» and the «house of darkness» represent two fundamentally opposed, causal principles of reality and its constitutive elements. The light and darkness imagery cannot, therefore, simply be taken as an element of dualism in the text.

Nondimeno, a dispetto di quanto vuol sostenere Popović sulla base di Albani, ${ }^{33}$ la «Dottrina dei Due Spiriti» ci è pervenuta e tale dato permette di contestualizzare più correttamente 4Q186 rispetto a chi sembra ritenere che la presenza di più documenti costituisca un problema anziché un'opportunità. La connessione fra i due testi in merito ai concetti di «luce» e «tenebra» è resa stringente, come si vedrà, dall'impiego accessorio delle metafore «residenza/ casa» e «sorgente/cisterna». Non ha quindi senso negare la presenza del dualismo in 4Q186.

Ancora, Popović obietta ad Alexander che questi non fornirebbe spiegazioni per il formarsi dei valori numerici delle parti di luce e di tenebra.

Ora il testo di 4Q186 recita, dopo il calcolo numerico (1, II, 8-9):

$$
\text { 8וזה הואה המולד אשר הואה ילוד עליו בברגל השור }
$$

e questa è la nascita [se usiamo la resa più ovvia per il termine, sotto la

\footnotetext{
${ }^{31}$ BRoOKe, «Body Parts», pp. 87-88.

32 Popović, Reading the Human Body, p. 190.

${ }^{33}$ Albani, «Horoscopes in the Qumran Scrolls», pp. 300, 312-313.
} 
quale egli è stato generato: nel piede del toro.

È da ritenere giusta l'interpretazione di Schmidt che vede nel numero nove i periodi diurni e notturni della gestazione, anche se non ne condivido l'opinione che vede in 4Q186 il meccanismo per determinare l'oroscopo sulla base del concepimento (מולד è un termine per il quale troppo ci si è spesi nel negarne il significato naturale). Il segno zodiacale è invece utile per definire le parti di luce e di tenebra procedendo a ritroso sulla base sicura della data di nascita e seguendo lo schema del cerchio zodiacale di Schmidt. Del resto, la stessa successione del testo suggerisce un significato del genere: «egli ha uno spirito nella casa della luce (per) sei (parti) e (per) tre (parti) nella casa della tenebra; poiché questo è (il momento della) nascita nel quale egli fu generato: nel piede del toro»: il perno della valutazione è fornito dal momento in cui l'individuo fu generato, non da quello in cui fu concepito.

Il terzo appunto che Popović muove ad Alexander è che questi non spiegherebbe adeguatamente l'espressione «nel piede del toro». Invero, si tratta di un appunto che non inficia il senso generale di quanto afferma Alexander dal momento, come si è già ricordato, ch'egli parla di «testo di fisiognomica astrologica», ove la menzione del toro si pone in riferimento al segno zodiacale.

Sorprendentemente, infine, Popović (pp. 287-288) non dedica molto spazio alle osservazioni di Brooke; in particolare, non si sofferma sulla summenzionata non banale interpretazione di אותות: sulla questione, tutt'altro che secondaria, avremo modo di tornare tra breve.

È a questo punto opportuno prendere in esame la «Dottrina dei Due Spiriti» nella sezione in cui vengono descritti i compiti dell'Istruttore (il Maskil), ossia in 1 QS III, 13-15: ${ }^{34}$

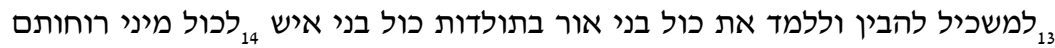

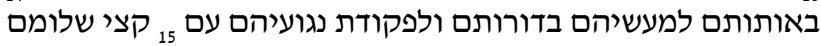

${ }_{13}$ Per l'Istruttore, affinché istruisca e renda edotti tutti i figli della luce sulla storia di tutti i figli dell'uomo ${ }_{14}$ riguardo le loro opere nelle loro generazioni e riguardo la visita del suo castigo e ${ }_{15}$ il tempo della sua ricompensa.

La struttura di questo passo è agevolmente comprensibile sulla base dell'utilizzo della preposizione ל. ${ }^{35}$ In particolare prendendo spunto dall'indagine strut-

\footnotetext{
${ }^{34}$ Per il testo di $1 \mathrm{QS}$, mi baso su C. MARTONE, La «Regola della Comunità». Edizione critica (Torino 1995).

${ }^{35}$ Sulla struttura grammaticale determinata dall'utilizzo di $ל$ in $1 \mathrm{QS}$, cfr. P. von OsteNSACKEN, Gott und Belial: Traditionsgeschichtliche Untersuchungen zum Dualismus in den Texten 
turale che ne è stata fatta da A. Lange, ${ }^{36}$ si possono schematizzare $\mathrm{i}$ periodi in una suddivisione di tipo A, B, C, D:

A) (È compito) del Maskil far conoscere e rendere edotti tutti i Figli della Luce sulla natura di tutti gli uomini:

B) secondo le tipologie dei loro spiriti (riconoscibili) nei loro segni,

C) secondo le loro azioni (riconoscibili) nelle loro generazioni ${ }^{37}$

D) e secondo la visita del loro castigo assieme alla definizione della loro ricompensa

In A -ove viene introdotta la mansione dell'Istruttore- la ha funzione specificativa, mentre nei tre che seguono sono esposti i criteri valutativi per definire la natura degli uomini. La connessione tra $\mathrm{i}$ periodi $\mathrm{B}, \mathrm{C}, \mathrm{D}$ è stilisticamente espressa dall'uso dell'endiadi tra B e C e della congiunzione all'inizio di C, ב che conclude l'esposizione. All'interno dei periodi B e C, la preposizione serve a introdurre le modalità utilizzate per comprendere le «tipologie» degli spiriti e le azioni degli uomini, ${ }^{38}$ mentre il periodo $\mathrm{D}$ si basa su quello che sarà il

aus Qumran (Göttingen 1969), pp. 537-538; in generale a Qumran: J. DuHaIME, «L'Instruction sur les deux esprits et les interpolations dualistes à Qoumrân (1QS III, 13-IV, 26)», RB 84 (1977), pp. 566- 594; in relazione a $1 Q S$ IV, 2-14: ID., «Les voies des deux esprits (1QS iv 2-14): "Une analyse structurelle"», $R Q$ 19/75 (2000), pp. 349-367.

${ }^{36}$ A. LANGE, Weisheit und Prädestination. Weisheitliche Urordnung und Prädestination in den Textfunden von Qumran (Leiden - New York - Köln), pp. 148-149: a) לכול מיני רוחותם באותותם,

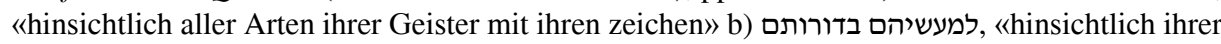
Taten in ihren Generationen»c) ולפקודת נגיעהם עם קצי שלומם, «und hinsichtlich der Heimsuchung durch ihre Plagen gemeinsam mit der Epochen des Heils». Lo schema di Lange è stato ripreso da J. Duнaime, «Cohérence structurelle et tensions internes dans l'Instruction sur les deux esprits (1QS III 13-IV 26)», in F. García Martínez (ed.), Wisdom and Apocalypticism in the Dead Sea Scrolls and in the Biblical Tradition (Leuven 2003), pp. 103-131: 107. Alcuni dubbi su questo schema sono stati avanzati da E. J. C. TIGChELAAR («'These are the names of the spirits of...': A Preliminary Edition of 4QCatalogue of Spirits (4Q230) and New Manuscript Evidence for the Two Spirits Treatise (4Q257 and 1Q29a)», RQ 21/4 [2004], pp. 529-547: 541-542): questi ritiene che 4Q255 e 4Q257 conservino parte del «Trattato dei Due Spiriti» di 1QS e di conseguenza osserva che le piccole varianti dei manoscritti relative alle espressioni dovrebbero indurci a cautela nella valutazione dei dettagli di un singolo manoscritto. Si deve tuttavia osservare che 4Q255 e 4Q257 potrebbero essere in questo caso portatori di innovazioni rispetto al testo di 1QS, senza contare che la scelta testuale del copista di quest'ultimo sia stata dettata dalla volontà di stabilire lo schema che si è individuato.

תולדה Secondo l'accezione di $\operatorname{Pr} 30: 11-14$, Sal 12:8, 14:5, 24:6, 73:15, 112:2, ove il termine serve a definire una disposizione d'animo.

${ }^{38}$ Secondo Lange, Weisheit und Prädestination, p. 148, la ב avrebbe invece funzione di avverbio temporale. 
risultato finale dell'esame degli spiriti: le misure del castigo e della ricompensa. Per quanto riguarda il termine תולדות, ritengo sia da accogliere l'opinione di G. Scholem, che propose la traduzione con «natura»: ${ }^{39}$ a sostegno ulteriore, si deve osservare che il sostantivo non può essere tradotto con «generazioni», concetto che si rivelerebbe incongruo all'interno del discorso, che verte sulla definizione di spiriti, azioni e retribuzioni escatologiche. Ma è il periodo $\mathrm{B}$, ove si prescrive di esaminare gli «spiriti» (רוחות) degli uomini per mezzo dei loro «segni» (אותות), che si rivela particolarmente interessante.

Ho già ricordato che Alexander ${ }^{40}$ e Brooke $^{41}$ identificano in questi «segni» $\mathrm{i}$ tratti fisici che riflettono lo stato spirituale di una persona ed entrambi ammettono un legame tra la concezione delle «parti di spirito» espressa in 4Q186 e la «Dottrina dei Due Spiriti» di 1QS III, 13-15. ${ }^{42}$ Non abbastanza, tuttavia, è stata evidenziata la portata del ricorrere dei «segni» (אותות) in entrambi i testi; Alexander vuole addirittura prendere le distanze da un accostamento preciso: «The «signs» ('otot) actually mentioned in the sermon [scil. la «Dottrina dei Due Spiriti»] as evidence of a person's participation in light and darkness are virtues and vices, such as

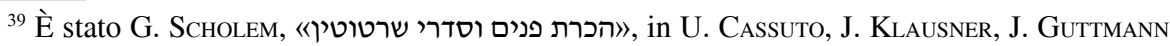

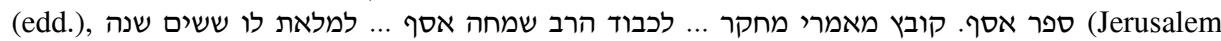
1953), pp. 459-495: 477-479, ad avanzare l'ipotesi che il significato di «natura» fosse da riferire all'utilizzo di תולדה in 1QS. Scholem prese le mosse dalla Fisiognomica di Rabbi Ishmael, ove si distinguono le תולדות dei giusti e dei malvagi: l'accezione del termine vi figura come terminus technicus che trae il proprio significato direttamente dalla corrispondente radice ילד come avviene

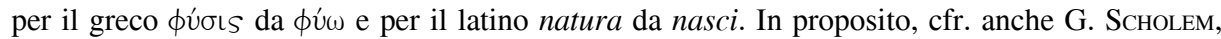
«Ein Fragment zur Physiognomik und Chiromantik aus der Tradition der Spätanticken jüdischen Esoterik», in AA.VV., Liber amicorum. Studies in Honour of Professor Dr. C.J. Bleeker (Leiden 1969), pp. 173-193: 180; l'accezione è stata accolta da ALEXANDER, «Physiognomy, Initiation, and Rank», pp. 390-391 e DAVILA, «4QMess Ar (4Q534)», pp. 369-370. Di diverso avviso J. Licht, «An Analysis of the Treatise of the Two Spirits in DSD», in Ch. Rabin, Y. YAdIN (eds.), Aspects of the Dead Sea Scrolls (Jerusalem ${ }^{2}$ 1965), pp. 88-100: 89-90 e J. DuhaIme, «Dualistic Reworking in the Scrolls from Qumran», $C B Q 49$ (1987), pp. 32-56: 40; P. ScHÄFER, «Ein neues Fragment zur Metoskopie und Chiromantik», in ID., Hekhalot Studien (Tübingen 1988), pp. 84-95: 91-92, n. 31 , ha ritenuto invece azzardata («gewagt») questa interpretazione, mentre il tradizionale senso di 'genealogia, discendenza' sarebbe sufficiente a esprimere la procedura di determinazione del fato di un uomo per mezzo delle caratteristiche fisiche.

${ }^{40}$ Alexander, «Physiognomy, Initiation, and Rank», p. 390.

${ }^{41}$ BrooKe, «Body Parts», pp. 87-88.

42 AleXAnder, «Physiognomy, Initiation, and Rank», pp. 390-391. Cfr. anche J. Licht, שוקיים סימן לבחירה 18; Stegemann, «Zu Textbestand», pp. 117-119; SсhмidT, «Astrologie juive ancienne», p. 139; la relazione tra i due testi è stata negata da R. BERGMEIER, Die Essener-Berichte des Flavius Josephus. Quellenstudien zu den Essenertexten im Werk des jüdischen Historiographen (Kampen 1993), pp. 52-53. 
humility, charity, greed and deceit. The declared principle seems to be «by their fruits shall you know them». But the language is highly suggestive and the possibility cannot be ruled out that those signs may have been extended to include physiognomic criteria». ${ }^{43}$

Tuttaria, è proprio all'interno del brano comunemente definito «Dottrina dei Due Spiriti» che si spiega il significato di רוח לו 4Q186. In 1QS, III, 13-14 si legge:

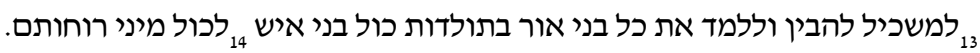

${ }_{13}(\grave{E}$ compito) del Maskil far conoscere e rendere edotti tutti i Figli della Luce sulla natura di tutti gli uomini ${ }_{14}$ secondo le tipologie dei loro spiriti.

Ora, l'impiego di רוח לו 4Q186 serve, attraverso la forma indeterminata, a richiamare non già direttamente lo spirito dell'esaminando, bensì la tipologia di tale spirito: la ripartizione delle parti di luce e di tenebra hanno questo fine. Questa precisazione, ritengo, è significativamente esplicativa dell'impiego del sintagma רוח לו 4Q186 e al contempo stabilisce un netto collegamento con la «dottrina dei Due Spiriti». Vi è un dato ulteriore, tutt'altro che secondario, utile a confermare siffatto collegamento. J. M. Allegro, che curò l'editio princeps del manoscritto, ${ }^{44}$ lesse come segue in 1, II, 7-8:

7 רוח לו בבית האור שש ושלוש בבור החושך

${ }_{7}$ Uno spirito per lui: nella casa della luce sei (parti) e tre nel pozzo ${ }_{8}$ delle tenebre

La lettura riguarda uno dei termini in scrittura «criptica» presenti nel manoscritto: BB' ’, ove convivono l'alfabeto greco (BB), quello ebraico ( ) e un segno dalla difficile interpretazione ('). Questa lettura ha destato perplessità tra gli studiosi, i quali in maggioranza hanno preferito emendare la lettura di Allegro in בבית, che peraltro si uniformerebbe alla terminologia impiegata più oltre nello stesso manoscritto - 1, III, 5-6. La lettura della yod sarebbe giustificata dalla sua supposta somiglianza alla waw presente in 4Q186, mentre rimane in questione l'interpretazione dell'ultimo segno. La lettura come taw dell'alfabeto fenicio richiede che del segno sia qui presente la sola parte sinistra della croce formata dalle due linee nella forma $\times$. Tuttavia, si deve osservare che il manoscritto non

${ }^{43}$ AleXAnder, «Physiognomy, Initiation, and Rank», p. 390.

44 J. M. Allegro, «An Astrological Cryptic Document from Qumran», JSS 9 (1964), pp. 291294; ID., Qumran Cave 4.I (4Q158-4Q186) (Oxford 1968), pp. 88-89. 
presenta danneggiamenti che giustifichino l'assenza della parte destra del segno e, d'altro lato, quest'ultimo presenta un ductus più morbidamente arrotondato rispetto alla stessa parte del medesimo segno ove esso ricorre in $4 \mathrm{Q} 186 .{ }^{45}$ Un'alternativa potrebbe essere quella di intendere il segno come la risultante di una voluta modificazione finalizzata a rendere ancora più criptica la lettura: ad esempio, una sorta di reš rimpicciolita e mancante di una parte del «tetto». Un meccanismo analogo è apprezzabile nel בבית che precede poco prima e che è scritto nella forma $\mathrm{BB} \angle \times$, ove la yod fenicia è mancante dei due trattini superiori orizzontali. Ci si potrà chiedere quale sia stato il senso, in quest'ultimo caso, di crittare una lettera che in 4Q186 1, III, 5 è invece riprodotta correttamente. Si può osservare in proposito da un lato che i criteri di crittazione non ci sono comunque noti; dall'altro, che questo manoscritto costituisce chiaramente una raccolta di vari esami che erano stati precedentemente redatti in forma individuale e non sempre seguendo uno schema stilisticamente precostituito: fa fede in tal senso l'utilizzo della preposizione מבית nel, di contro ai בבית di 1, II, 7 e 1, III, 5. Il copista, si deve ascriverglielo a merito, non operò un'arbitraria normalizzazione stilistica, ma intese riprodurre fedelmente i singoli antigrafi nelle loro peculiarità grammaticali e grafiche.

Un'altra riflessione è opportuna in merito alla differenziazione tra yod e waw. In 1, III, 5 disponiamo di un eloquente confronto tra le due lettere nella parola רגליו: la prima mostra un angolo più arrotondato e relativamente ampio tra il «tetto» e la linea orizzontale; la seconda, al contrario, ha un angolo più netto e acuto. Rispetto a tali caratteristiche, la nostra parola criptata mostra di contenere una waw anziché una yod. Sulla base di queste osservazioni la lettura da prediligere è di conseguenza בבור.

A questo punto, è opportuno soffermarsi su quanto degli spiriti viene detto in 1QS III, 17-19 e in 4Q186 II, 7-8:

1QS III, 17-19

17 ... והוא ברא אנוש לממשלת תבל וישם לו שתי שתי רוחות להתהלך בם עד מועד פקודתו

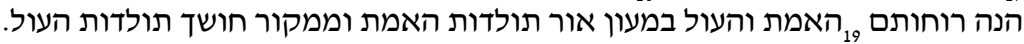

17...Egli creò l'uomo per sovrintendere ${ }_{18}$ al mondo e pose in lui due spiriti affinché egli procedesse secondo la loro conformità fino al momento della Sua visita. Essi sono gli spiriti ${ }_{19}$ di verità e di ingiustizia. Nella residenza ${ }^{46}$

\footnotetext{
${ }^{45}$ Sono grato, per l'interpretazione di questo segno, a Corrado Martone (Università di Torino) per le sue osservazioni di carattere paleografico.

${ }^{46}$ Alcuni (tra questi lo stesso AleXANDER, «Physiognomy, Initiation, and Rank») leggono מעין,
} 
di luce si trova la natura della verità e dalla sorgente di tenebra proviene la natura dell'ingiustizia.

$$
\text { 4Q186 II, 7-8 }
$$

7רוח לו בבית האור שש ושלוש בבור החושך וזה הואה ומולד אשר הואה ילוד עליו.

${ }_{7}$...egli [scil. l'esaminato] ha sei parti nella casa della luce e tre parti nella cisterna ${ }_{8}$ della tenebra.

Alexander afferma ${ }^{47}$ esservi un richiamo tra i rispettivi usi di מעון / מער / בור / מקור. In effetti, in questi passi i riferimenti concettuali a una residenza/ casa della luce e a una sorgente/cisterna della tenebra veicolano un legame semantico significativo per poter dire che vi si parla della stessa cosa: in altri termini, il rapporto tra i due testi è stabilito e rafforzato dall'utilizzo di termini appartenenti da un lato al campo semantico delle abitazioni, dall'altro a quello delle acque. Se consideriamo poi le parole di 1QS III, 13-15 e segnatamente quelle del periodo B), si capisce cosa si intenda quando si vuole esaminare la natura degli uomini «secondo le tipologie dei loro spiriti (esaminati) per mezzo dei loro segni»: una volta trovato il collegamento con un testo fisiognomico, come nel caso in questione, $\mathrm{i}$ «segni» sono chiaramente da identificare con le caratteristiche fisiche. ${ }^{48}$

Di conseguenza, appare inappropriata la tesi di Popović, che vuol vede-

\footnotetext{
'sorgente', ma la forma della terza lettera -nonostante la ben nota somiglianza tra ) e ' a Qumran- è a mio parere indubitabilmente quello di una ו. D'altro lato, מעין è lettura arbitraria conseguente alla volontà di stabilire un parallelismo con מקור: cfr. DuhaImE, «Dualistic Reworking», p. 40.

${ }^{47}$ AleXANDER, «6. I rotoli del Mar Morto», p. 475; AleXAnder, «Physiognomy, Initiation, and Rank», p. 390.

${ }^{48}$ AleXANDER, «Physiognomy, Initiation, and Rank», p. 390. BRoOKe, «Body Parts», pp. 87-88, ha mostrato come $\mathrm{i}$ «segni» corrispondano effettivamente alle caratteristiche fisiche, mettendo in rilievo l'importanza che queste hanno all'interno degli inni 4Q434 e 4Q436, appartenenti al genere $B^{e} k \hat{\imath} \hat{\imath}$ Nafšl, specialmente se messi in relazione (pp. 85-86) con le norme di integrità fisica richiesta ai componenti della comunità, norme che sono esplicitamente esposte nella Regola della Congregazione (1Qsa II, 3-9), nella Regola della guerra (1QM VII, 4-5) e in una delle copie qumraniche del Documento di Damasco (CD XV, 15-17). Si deve aggiungere che una comprensione «fisiognomica» del testo di 1QS III, 13-15 rende più perspicuo anche quanto viene detto in IX, 12-16, sempre in riferimento alle norme che il Maskil deve osservare per la valutazione degli uomini (sulla relazione tra la figura del Maskil e i testi fisiognomici di Qumran si sono favorevolemente espressi: ALEXANDER, «Physiognomy, Initiation, and Rank», p. 391; Albani, «Horoscopes», p. 320; Brooke, «Body Parts», pp. 87-88). AleXander (p. 390), che pure ha individuato il nesso di 1QS con il testo fisiognomico 4Q186, ha preferito non stabilire questa corrispondenza, assimilando $\mathrm{i}$ «segni» a sentimenti come l'umiltà e la carità da un lato, la cupidigia e la falsità dall'altro.
} 
re, in connessione con il toro, il riferimento a uno «spirito» animale esterno all'uomo. Si può fare poi una considerazione ulteriore in merito al «piede» del Toro. Questo è da considerare un dettaglio dal preciso significato all'interno di una rappresentazione figurata dello zodiaco: nell'ultima decade del segno il pianeta Mercurio incontra il passaggio del sole nella costellazione ed è da intendere che la posizione di quest'incontro si trova all'interno della zampa anteriore dell'animale. Un parallelo significativo di questo tipo di congiunzioni è testimoniato nei testi astrologici babilonesi; ad esempio in un testo del capo scriba Issar-šumu-ereš (VIII sec. a.C.) è dato leggere: «Quando Marte, inoltre, retrocede dalla testa [il corsivo è mio] di Leone e tocca Cancro e Gemelli, la sua interpretazione è la seguente: Fine del regno del re del paese occidentale». ${ }^{49}$ Questa dipendenza dalla tradizione babilonese, inoltre, ci aiuta a definire anche il carattere della «pietra di granito» che viene menzionata in 4Q186. ${ }^{50}$ In due elenchi babilonesi delle costellazioni zodiacali, ciascun segno è accompagnato dal «nome di un tempio o di una città, seguiti dalla menzione di un albero, di una pianta e di una pietra [il corsivo è mio], più o meno preziosa». ${ }^{51}$

È vero, a questo punto, che il confronto tra la descrizione degli Esseni fornita da Flavio Giuseppe e il testo della Regola della Comunità condividono un elemento che è stato possibile individuare grazie all'esame di un manoscritto ulteriore, ma v'è un dato in più, se si procede a indagare i dettagli: se da un lato sia in Flavio Giuseppe che nella Regola si parla dell'uso della fisiognomica, è d'altro lato il modo in cui se ne parla che deve farci riflettere. Si tratta di un modo nascosto, che in Flavio Giuseppe poté esser tale per il rispetto portato ad alcune notizie acquisite ma sulle quali si doveva mantenere il riserbo; nel caso della Regola, il modo elusivo di accennare all'argomento rispondeva alla necessità di mantenere lo stesso riserbo, stavolta in seno alla comunità stessa, ove evidentemente era 1'Istruttore stesso

${ }^{49}$ Cfr. G. Pettinato, La scrittura celeste. La nascita dell'astrologia in Mesopotamia (Milano 1998), p. 206; per l'importanza dell'influsso dei pianeti sulle costellazioni, ID., pp. 115-116; sulla questione in generale si veda, con ampia bibliografia, A. AABOE, «Babylonian Mathematics, Astrology, and Astronomy», in J. Boardman, I. E. S. Edwards, N. G. L. Hammond, E. Sollberger (Eds.), The Cambridge Ancient History. III.2. The Assyrian and Babylonian Empires and other States of the Near East, from the Eighth to the Sixth Centuries B.C. (Cambridge $\left.{ }^{2} 1991\right)$, pp. 276292.

${ }^{50}$ M. Popović, «Physiognomic Knowledge in Qumran and Babylonia: Form, Interdisciplinarity, and Secrecy», Dead Sea Discoveries 13 (2006), pp. 150-176: 171.

${ }^{51}$ Petrinato, La scrittura celeste, pp. 114-115, ove sono riportati gli elenchi, tratti da E. F. WEIDNER, Gestirndarstellungen auf babylonischen Tontafeln (Wien - Graz 1967), pp. 17-18. 
a padroneggiare i dettagli dell'esame fisiognomico; è d'altro lato evidente che l'accidentale conoscenza delle norme di analisi da parte di coloro che ambivano all'ammissione avrebbe potuto falsare la prova. Che questo desiderio di segretezza fosse effettivo e reale viene infine confermato dalla composizione «criptica» di 4Q186. ${ }^{52}$

Recibido: $18 / 08 / 2009$

Aceptado: 26/04/2010

${ }^{52}$ Alexander, «6. I rotoli del Mar Morto», p. 475; Albani, «Horoscopes», p. 372. 
